\title{
Lipid Review and Teaching Tips in Nutrition
}

\author{
Kathleen Schmalz and Pamela K. Kerrigan \\ College of Mount Saint Vincent
}

\begin{abstract}
This paper reviews lipid structure, function, digestion, absorption, and metabolism. Tips to teach this subject area in college-level health education courses are offered along with the review.
\end{abstract}

(C) 2003 Californian Journal of Health Promotion. All rights reserved.

Keywords: lipids, fats, teaching health science, nutrition

Lipid Review and Teaching Tips in Nutrition

\section{Introduction}

This paper will consider methods of teaching college students to apply a scientific framework to the study of nutrition. It will highlight the dynamics of scientific inquiry and reveal how it may be utilized to analyze the role of nutrition in health. Encouraging students to take an active hands-on approach to learning about the science of fat content within foods today, this study promotes students the creation of diagrams, comparisons of various foodstuffs, and personal goal setting in terms of diet and nutrition.

It is important to first stress that fat exists as an essential nutrient in food. It is a primary source of energy and also yields significant levels of both taste and texture to food. Fat is an important component of cell membranes, and in the biosynthesis of significant regulatory molecules. As with other vital nutrients, there are deficiencies when a diet has too little fat but an overabundance of fat in the diet significantly perpetuates the risk of chronic disease (Goldman, 1994)

Fat denotes a natural oily or greasy substance that is contained within animal bodies. Fats are construed to be solid in texture, in contrast to oils, which remain in a liquid state at ambient temperatures. On a scientific level, there is little difference between a fat and an oil, but they are chemically unlike the oils utilized in the petroleum industry known as hydrocarbons (Jeffreys, 1991).

The more general term lipid is used to designate a chemically diverse group of fatty substances, which are poorly soluble or insoluble in water, but soluble in nonpolar organic solvents such as chloroform, hydrocarbons, alcohols or ethers. Lipids are comprised of carbon, hydrogen, and oxygen (sometimes nitrogen and phosphorous) in proportions that contrast with those of other nutrients. This class of biomolecules include fatty acids, fatty acid derivatives, sterols and its derivatives, the fat-soluble vitamins $\mathrm{A}, \mathrm{D}$, $\mathrm{E}$, and $\mathrm{K}$, and the prostaglandins.

The triglycerides, which are composed of a foundational 3-carbon molecule glycerol and three fatty acids, are one of the major classes of lipids that exist in the human body (Figure 1). 


\section{Triglyceride}

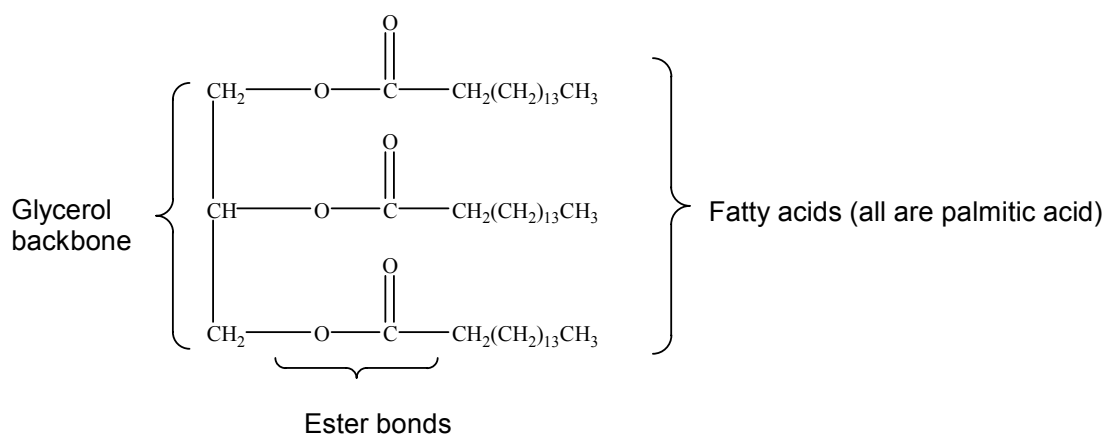

Figure 1

Triglyceride molecular structure

The general structural unit of a fatty acid is a long hydrocarbon chain with an acid, or carboxyl, group at one end (Figure 2). The carbon chains in these fatty acids differ in length from a few to 20 or more carbons (Johnson \& Sanders, 1994). Short chain fatty acids range from 4 to 7 carbons in length and these remain liquid at colder temperatures. A good example for the student to visualize is that the fatty acids within half-and-half remain liquid even in the refrigerator. Medium chain fatty acids, such as those in coconut oil, range from 8 to 12 carbons. They become solid within the refrigerator but remain liquid at room temperature. Extended chain fatty acids, exceeding 12 carbons, such as those in venison fat, typically remain solid at room temperature. The majority of fatty acids within animals, including humans, and plants, contain anywhere from 14 and 22 carbons.

Saturated fatty acids contain only carboncarbon single bonds (Figure 3) in their chain. The most prevalent saturated fatty acids are palmitic acid, with 16 carbons, and stearic acid, with 18 carbons. They are found most frequently in animal products. Vegetable derivatives of saturated fatty acids include palm oil, palm kernel oil, and coconut oil which are plants that are common in tropical climates (Johnson \& Sanders, 1994).

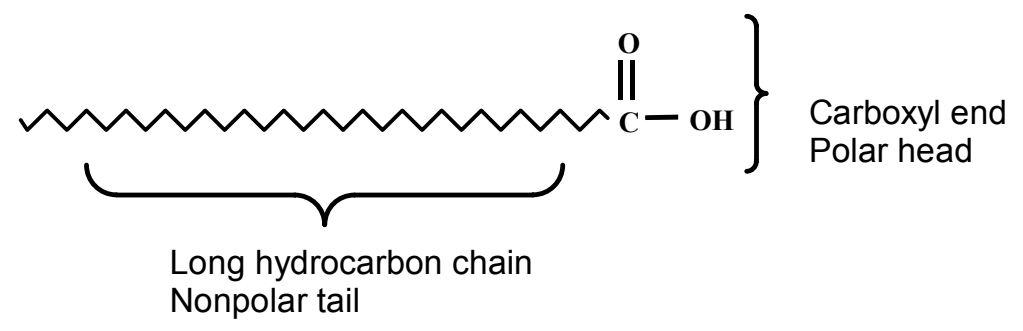

Figure 2

General Molecular Structure of a Lipid 


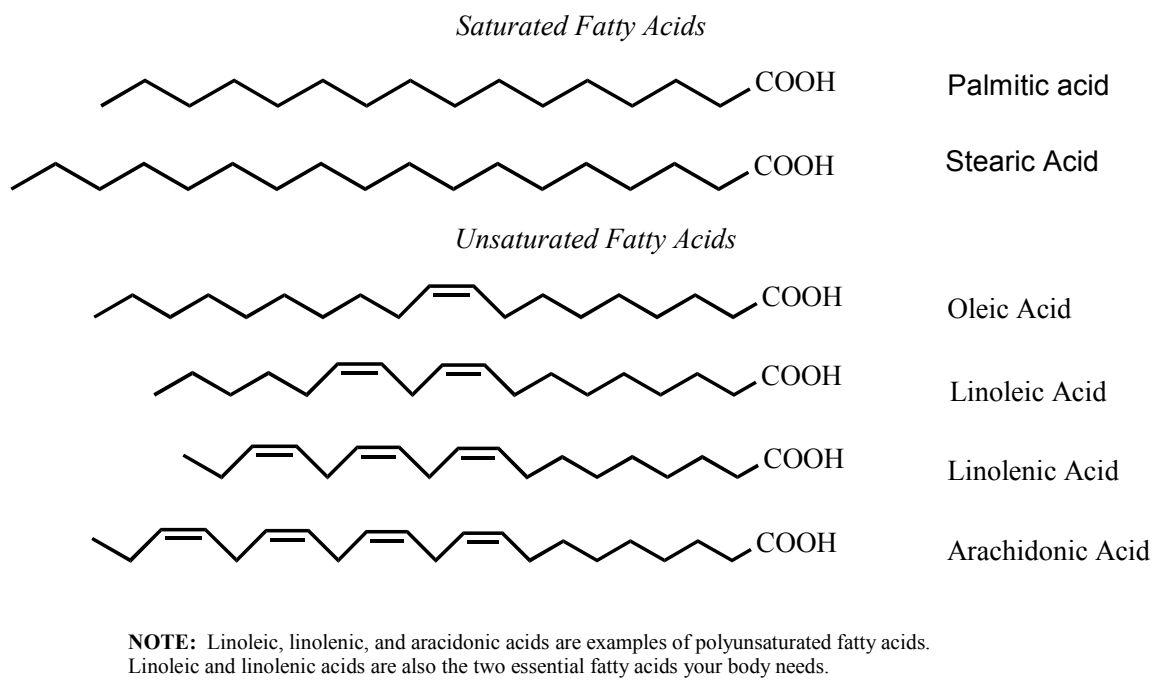

Figure 3

Molecular Structure of Saturated and Unsaturated Fats

An unsaturated fatty acid contains carboncarbon double bonds (Figure 3). The fatty acids containing one double bond are monounsaturated fatty acids (e.g., oleic acid, found in olive and canola oils). Those that contain more than one double bond are called polyunsaturated fatty acids (e.g. linoleic acid, found in corn, safflower, and soybean oils). Unsaturated fatty acids are generally liquid at cooler temperatures. The more unsaturated a fatty acid is, the more likely it is to exist as a liquid at room temperature. The majority of fatty substances in our diets contain various amounts of saturated, monounsaturated, and polyunsaturated fatty acids. Omega-3 and omega- 6 fatty acids are precursors of compounds called eicosanoids that assist in controlling both blood pressure and clotting, the immune function, and additional body dynamics.

Important to stress when teaching students about fat composition is the fact that it is the type of fatty acids in triglycerides that determines their texture, taste, and physical aspects. The triglycerides contained within pork possess predominantly long chain saturated fatty acids - consequently, the fat on a porkchop remains solid at room temperature. The amounts and kinds of fatty acids in a Hershey's bar, for example, enable it to remain solid at room temperature, but to melt rapidly and efficiently within the mouth (Goldman, 1994).

It is important to emphasize to students that triglycerides may be utilized as an instantaneous source of power or stored in the adipose tissue for later use within the body. Since triglycerides exist as a concentrated energy derivative, a substantial degree of energy can be stored without a large augmentation in corporeal mass.

Phosphoglycerides, being similar to triglycerides, possess the same foundation of glycerol but they contain only 2 fatty acids. Instead of the third fatty acid there is a phosphate group (Figure 4), which is connected to another alcohol. The fatty acid end is soluble in fat, whereas the phosphate end is water-soluble. This construction permits phosphoglycerides to enter into water and fat, an attribute that renders them vital for many functions in the food supply and the body. 


\section{Phosphoglyceride}

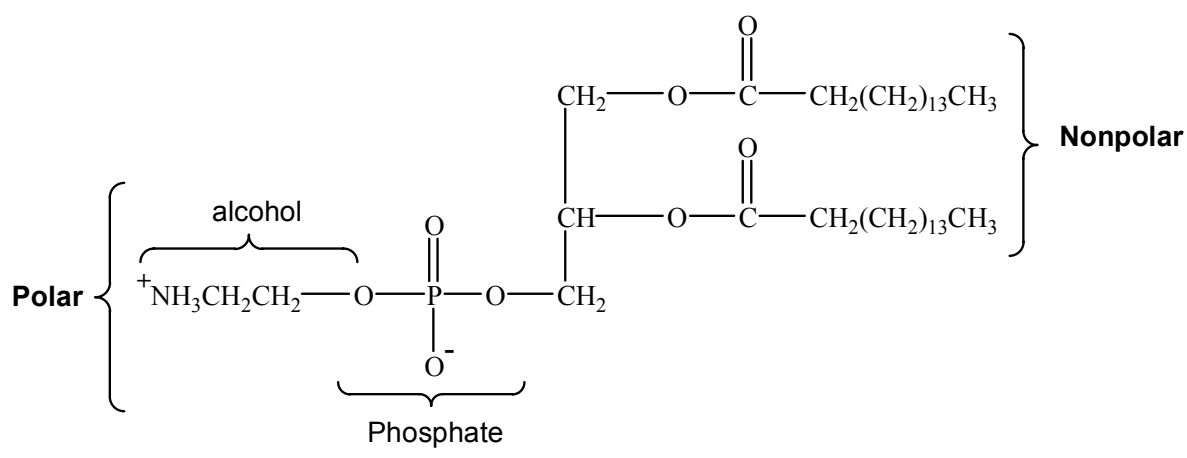

Figure 4

Molecular Structure of a Phosphogylceride

A fourth class of lipids is the sterols (Figure 5) composed of several ring-like forms. These are unlike triglycerides and phosphoglycerides in that these do not dissolve in water. Cholesterol, the most important sterol, is found only in foods derived from animal sources. Egg yolks and organ meats such as liver and kidneys are rich in this steroid. For instance, a single egg yolk may contain up to $216 \mathrm{mg}$ of cholesterol. Liver possesses about 350 $\mathrm{mg}$ per 3.5-ounce serving. Less fatty red meats and skinless chicken, by contrast, have no more than $100 \mathrm{mg}$ (Johnson \& Sanders, 1994).

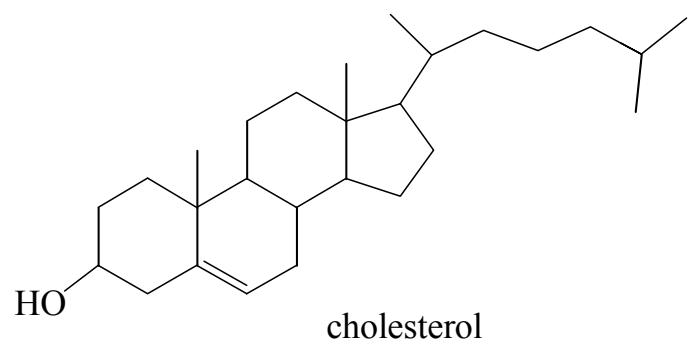

Figure 5

Molecular Structure of Cholesterol

Crucial to understanding fats are the methods by which lipids are both digested and absorbed in the body. A limited degree of lipid digestion commences in the stomach in response to the action of lipases, lingual (mouth) and gastric (stomach). These work mainly upon triglycerides containing short and medium chain fatty acids such as those in milk, and so are especially important in young children. In the average adult, the digestion of dietary fat primarily occurs within the small intestine due to the action of lipases released by the pancreas. A helpful learning mechanism for students involves their completing an anatomical overview of lipid digestion in diagram form. Students then delineate the absorption process: bile, fabricated by the liver helps emulsify fat in order to aid its digestion in the small intestine; end, via process in the large intestine.

Once lipids are digested and absorbed, they are available for utilization by various tissues of the body, to where they are delivered via lipoproteins. When required for cell functions, lipoprotein lipase, which 
is connected to capillary and cell membranes, relinquishes the lipid portion from its protein carrier and hydrolyzes the triglyceride into glycerol and fatty acids. The metabolic requirements of the organism identify the specific metabolic dynamic, which will occur, such as lipolysis (a method of fat dissipation) lipogenesis, or fat synthesis, each involving different pathways, enzymes, and reactants (Jeffreys, 1991).

Students should understand that the constituent cells of the liver encompass a complicated internal biochemistry laboratory involving a myriad of different processes. Fatty acids are joined within the liver by lipogenesis into new triglycerides. These are typically carried exterior to the liver by the lipoproteins, particularly the VLDL's, that transport them to adipose tissue for storage until needed. Lipotropic components, such as choline and methionine instigate lipogenesis and subsequent transport; this prevents excessive accumulation in the liver. Fatty acids and glycerol brought together from carbohydrates travel the identical pathway as the triglycerides synthesized directly from digested lipid. Overabundant accumulation of kilocalories from carbohydrates augments our storage of fatty substance.

Students should note that lipolysis is taking place within the liver, as triglycerides are hydrolyzed to create fatty acids and glycerol. The triglyceride reactions are reversible and take place in answer to the requirements of the organism. When there is an overabundance of lipid within the liver, lipogenesis will transform it into a state conducive to both transport and storage. In instances when an individual's energy needs demand accessible lipid, lipolysis will create available lipid products for circulation. Triglycerides can be hydrolyzed, resynthesized, and utilized as a source of energy, or employed in the synthesis of additional lipids, such as phospholipids and cholesterol. In order to master the intricacies of this complex process, students will complete a diagram that delineates the manner in which triglycerides produce energy. Contrasts may be drawn between glycerol, a 3-carbon molecule that can be converted into pyruvate, and fatty acids, which break into 2 -carbon units and enter the citric acid cycle as acetyl-CoA (Goldman, 1994).

Adipose tissue, a source of contained energy, is stored in the abdominal cavity and muscle tissue, where it is metabolically active. It protects the body from fluctuations in external environment and creates a shock guard cushion.

The health implications of food fat content are vital to college-level investigations of nutrition science. Genetic predisposition, dietary content, and other environmental aspects contribute to the development of cardiovascular problems, obesity, cancer, and a myriad of other diseases of humans (Smith, 1993). By improving one's diet composition one may retard the rate of disease development. Cholesterol, however, is a necessary compound of every living animal cell and consequently is a natural constituent in foods obtained from animals. Despite the fact that the effect of typical dietary cholesterol intake on blood cholesterol concentration in humans is sometimes minimal, the relationship of blood cholesterol to the development of cardiovascular disease has concerned consumers in their dietary cholesterol intake.

Intake of saturated fat is associated positively with blood cholesterol concentration, and thus saturated fats are considered a primary risk factor for heart disease. K.C. Hayes (1996) confirms that an acute interest in the metabolism of fats and oils developed as early as the 1950s, when it was initially determined that dietary fat saturation exerted a considerable influence upon the total plasma cholesterol concentration or TC (Hayes, 1996). Blood cholesterol concentrations diminish, as does 
the risk of heart complications, when polyunsaturated fats replace saturated fats. Current investigations have confirmed that all dietary saturated fatty acids do not identically affect blood cholesterol concentration in human beings. A substance such as palmitic acid is proven to augment cholesterol concentrations, yet stearic and the shorter chain saturated fatty acids appear to cause no fluctuation in plasma cholesterol levels.

Cholesterol is mandatory for the body but it is not essential in the diet. Almost all of the cholesterol in the body can be found in cell membranes. It is also a part of myelin, the coating on many nerve cells. Cholesterol is required to synthesize vitamin $\mathrm{D}$ in the skin; cholic acid, a component of bile; and some hormones, such as testosterone and estrogen which propel growth and the development of gender characteristics; and cortisol, which instigates glucose synthesis in the liver (Jeffreys, 1991).

The diet of most Americans contains about equal ratios of fat from plant and animal sources. As a result of the association of food fats with a host of human illnesses, in particular cardiovascular disease, cancer and obesity, the agricultural and food industries are now promoting fresh and processed foods with less total fat, saturated fat and cholesterol so that consumers may follow current nutritional advice without changing the types of food they habitually consume. Even more research remains to be done, to clearly delineate the relationship between food fats and health, to develop technologies for making effective transformations in food composition, and to describe the physiological and biochemical mechanisms of the effect of food fats on health welfare.

The nearly 30-percent increase in our national fat intake since 1910 is related to three dietary factors - fats and oils, ice cream, and red meat. The consumption of specific types of fats - cooking fats such as lard and shortening combined has remained about the same, while the consumption of butter and margarine combined diminished, (Goldman, 1994). Especially significant are dramatic augmentations related to more sophisticated food technology and marketing.

Students will be given ample opportunity to study the contents of various food labels that provide a range of information on the fat content within food. This information allows one to identify sources of dietary fat. Once consumers understand how to utilize this information they can make more educated choices. The Ingredients List reveals the source of fat, such as corn oil or soybean oil, and the Nutrition Facts indicates the kcalories from fat, the grams of fat and saturated fat, and the milligrams of cholesterol in a single serving. These are revealed as a percent of the Daily Value. The Daily Values are determined for a diet containing 2000 kcalories and are based on the recommendation that the diet should not exceed greater than $30 \%$ of energy from fat, no more than $10 \%$ from saturated fat, and no more than $300 \mathrm{mg}$ of cholesterol per daily intake. The percent Daily Value permits consumers to determine in an instant how a particular food will fit into the recommendations for fat intake for the day. If a serving contains $50 \%$ of the Daily Value for fat, the remainder of the day's intake will have to be prudently selected to not exceed the recommended allowances.

Some food labels may be inscribed with phrases such as "fat free," "low cholesterol," or "lean." These terms reflect the manufacturers attempt to respond to the public's concern with lessening fat intake. To render these descriptors meaningful to consumers as well as to manufacturers, food-labeling regulations have assigned standard definitions for these terms. A product designated as "low fat" cannot exceed more than three grams of fat in a single serving. It should be stressed to students that these terms could be utilized only in manners that do not confuse consumers. Since saturated fat in the diet 
increases blood cholesterol levels, a food that is low in cholesterol but high in saturated fat, like crackers baked with coconut oil, cannot be deemed to contain "low cholesterol" content, since it may indeed serve to augment blood cholesterol in the body (Smith, 1993).

An aspect of food labels that may be complicated for consumers is the claim that a product is a particular percent fat free. This refers to percent by weight, not by energy. To prevent potential misunderstanding, labeling laws mandate that a product can profess to be a certain percent fat free only if it is additionally a fat-free or low fat food. For instance, low fat sausages that are labeled $97 \%$ fat free contain 1 to 1.5 grams of fat, which accounts for $10 \%$ of the total kcalories and accounts for $2 \%$ of the Daily Value. An eye-opening exercise for students can involve their matching up descriptors such as "fat free," "saturated fat free," "low saturated fat", "low cholesterol" with their precise definitions, such as:

"contains less than $1 / 2$ gram of fat per serving"

"contains less than $1 / 2$ gram of saturated fat per serving and less than $1 / 2$ gram trans fatty acids per serving"

"contains one gram or less of saturated fat and not more than $15 \%$ of kcalories from saturated fat per serving"

"contains less than $20 \mathrm{mg}$ of cholesterol and 2 grams or less of saturated fat per serving"

These subtle and respective differences confirms the potentially elusive nature of food labeling (Smith, 1993).

Manufacturers have begun to utilize many fat substitutes (carbohydrate based) that replicate the texture and taste of fat without the objectionable health ramifications as a means of lessening the fat and energy content within food. Various pectins and gums are frequently included in foods to approximate the viscosity and full-bodied aspect that fat creates. Many fat-free salad dressings are enhanced with gums. Many of these products are unstable when heated and experience a short shelf life. Indeed, most low fat and fat-free products, (such as various brands of fat-free mayonnaise, parmesan cheese, and pretzels) contain a combination of ingredients that offer the texture of fat. In many products, fats are substituted with other energy-containing nutrients, so the energy content of fat-free foods may not be significantly lessened. Fat-free products can assist in diminishing the amount of fat in the diet, but they cannot be eaten excessively without influencing overall energy intake.

An exercise students can perform to help them apply critical thinking skills to their own nutritional choices involves their calculating their own average fat, saturated fat, and cholesterol intake using a three-day record of the food they consume. Ideally this assignment should use a food consumption analysis software program, but when not available, results can be calculated by hand. Determining both the amounts of fat and saturated fat he or she has consumed, the student may be encouraged to consider ways of lessening total intake of saturated fat in the diet. Recommendations for decreasing fat intake include restricting amounts of high-fat foods and augmenting intake of foods high in complex carbohydrate and low in fat (Georgiou, 1993). In addition, he or she can investigate how calculated levels of cholesterol intake compare with recommended daily allowances.

It is important to introduce students to the controversy surrounding Olestra, nonabsorbable artificial fat made of sucrose with fatty acids linked to it. Used in specific snack foods, this substance offers the taste and texture of fat without its health dangers or kcalorie levels. As with other additives, the attractive features of Olestra must be measured against its risks. It has taken twenty five years to manufacture 
Olestra and gain FDA approval because various risks associated with this substance account for this extended period of time for development. It is the prevalence of fatty acids that gives Olestra its similarity to fat. The difference between this substance and normal triglycerides, is that either the human enzymes or the bacterial enzymes within the intestines cannot process Olestra, and it is expelled as waste without being absorbed into the body.

The primary problem with Olestra is that it lessens the absorption of various fat-soluble vitamins. To avoid diminishing the value of these vitamins, Olestra has been fortified with some vitamins but it has not been fortified with beta-carotene and other fatsoluble substances that may be vital for good health. An additional potential complication with this substance is that it can create gastrointestinal upset and diarrhea in some individuals. Olestra travels through the GI system with being digested, thus, draws water into the intestines. The FDA mandates a warning about these possible problems on the labels of products which purport to contain this product (Dunn, 1996).
When eaten in reasonable amounts, Olestra creates little problem for the majority of individuals. Olestra-containing products lessen fat intake without altering the virtual nature of the diet. For instance, if a snack of low-fat dip and high-fat chips is substituted with one of low-fat dip and Olestra-enhanced chips, fat intake will be diminished but flavor will not. However, if Olestra-enhanced products are consumed in such substantial amounts that they replace the consumption of grains, fruits, and vegetables, the consequence might be a diet low in fat but also lacking in fiber, vitamins, and minerals (Dunn, 1996).

This paper has sought to present various methodologies by which college students apply a scientific overview to nutritional analysis. By promoting an active approach to learning about the science of fat content within foods in the 1990s, this papers seeks to render the study of nutrition science more understandable and significant for undergraduates. Consequently we have included some possible review questions that are designed to encourage the students to think critically about the topic of lipids.

\section{References}

Dunn, T. (1996). A study of artificial food substitutes. Cambridge: Lindon Quarry Publications.

Georgiou, C. C. (1993). Saturated fat intake of elderly women reflects perceived changes in their intake of foods high in saturated fat and complex carbohydrate. Journal of the American Dietetic Association, 93, 1444-1445.

Goldman, H. (1994). Reducing overall fat intake. Baltimore: HTP Science Publications.

Hayes, K. C. (1996). Designing a cholesterol-removed fat blend for frying and baking. Food Technology, 4 , 92-95.

Jeffreys, R. (1991). Scientific food analysis. Oxford: Robertson Publications.

Johnson, P. T., and Sanders, C. (Eds.) (1994). Nutrition. Cincinnati: RNF Publications.

Smith, A. L. (1993). The American diet and disease. Cincinnati: Davies \& Sons Ltd.

Trexler, M. L., and Sargent, R. (1993). Assessment of nutrition risk knowledge and its relationship to the dietary practices of adolescents. Journal of Nutrition Education, 25, 337-342. 


\author{
Author Information \\ Dr. Kathleen Schmalz \\ 148 Forest Avenue Extension \\ Westwood, New Jersey 07675 \\ Ph. 201-664-0308 \\ E-mail: DrKSmagic@aol.com \\ Dr. Pamela K. Kerrigan \\ 120 N. Broadway \#13-D \\ Irvington, New York 10533 \\ Ph. 914-591-4549 \\ E-mail: Pkerriga@manhattan.edu
}

Kathleen Schmalz, EdD, RN, CHES is an Associate Chair, Department of Health and Human Services, Director Health Education Program and Pamela K. Kerrigan, $\mathrm{PhD}$ is an Associate Chair in the Joined Departments of Chemistry and Biochemistry at the College of Mount Saint Vincent, Riverdale, New York City, 10471.

Drs. Schmalz and Kerrigan have been assisting in the instruction of their respective areas in each other's courses. 


\section{Appendix A}

Sample Exam Questions

1. How does one differentiate between a saturated fat and a monounsaturated or polyunsaturated fat?

2. What is the most prevalent type of lipid in the body and in food ? (HINT: they are composed of 3 fatty acids on a glycerol backbone)

3. In order to enable transport in the body, lipids are associated with which substance as a means of creating lipoproteins?

4. Name a sterol that is created within the body and consumed in the diet.

5. Which tissue is stored beneath the skin and around body organs, and is composed of fat-storing cells?

6. Define a lipoprotein.

7. Which foodstuffs may be attributable to the increase of fat consumption by Americans during this century?

8. What is the function of fat within the human body?

9. Name two diseases associated with excessive cholesterol levels.

10. Will fatty foods containing a high proportion of saturated long-chain fatty acids are found in a liquid or solid state?

\section{Answers}

1. saturated fat refers to a fat or fatty acid in which the carbons are bound to as many hydrogens as possible \& therefore contains no carbon-carbon double bonds in its structure; monounsaturated fatty acid contains one carbon-carbon double bond; polyunsaturated fatty acid contains more than one carbon-carbon double bond

2. triglycerides

3. protein

4. cholesterol

5. adipose tissue

6. a substance containing a base of lipids surrounded by an outer casing of protein, phospholipid, and cholesterol

7. fats and oils; ice cream; red meat

8. fat derives its functional role as a source of energy and a factor in the synthesis of cholesterol and its derivatives as well as of lipids, prostaglandins, and other body substances

9. heart disease, cancer

10. solid 